Supporting Information for

\title{
Versatile Printing of Substantial Liquid Cells for Efficiently Imaging In-Situ Liquid-Phase Dynamics
}

Zhiwen Liu, Zetan Cao, Jia He, Haoran Zhang, Yujun Ge, Bin Chen*

Center for Ultrafast Science and Technology, School of Physics and Astronomy, and School of Chemistry and Chemical Engineering, Shanghai Jiao Tong University, Shanghai 200240, Republic of China

E-mail: cbcce@sjtu.edu.cn

\section{This file includes:}

Materials and Methods

Supplementary Notes

Figures S1 to S9

Captions for Movie S1 to S7 


\section{MATERIALS AND METHODS}

Synthesis of Au nanoparticles. The first step was to synthesize the Au seeds. $50 \mathrm{~mL}$ of $2.2 \mathrm{mM}$ sodium citrate aqueous solution was added to a three-neck round-bottom flask, which was heated till boiling. Then, $1 \mathrm{~mL}$ of $4.17 \mathrm{mM} \mathrm{HAuCl}_{4}$ solution was injected into the flask, followed by continuously stirring and heating for $15 \mathrm{~min}$. Finally, the Au seeds ( $\sim 15 \mathrm{~nm}$ in size) could be obtained. The mixture solution was kept at the temperature of $90{ }^{\circ} \mathrm{C}$ so that the reaction rate was reduced.

The Au nanoparticles (NPs) with the different sizes were prepared by repeating the following procedure. $1 \mathrm{~mL}$ of $4.17 \mathrm{mM} \mathrm{HAuCl} 4$ was again injected into the flask containing the Au seeds, followed by stirring at $90{ }^{\circ} \mathrm{C}$ for $30 \mathrm{~min}$. Normally, the Au NPs with $\sim 50 \mathrm{~nm}$ in size could be obtained after five repetitions, which was used in the liquid cells for the studies.

Versatile printing of substantial liquid cells for TEM characterization. The liquid cells were prepared by electrospinning in the core-shell mode (see Figure 1 in the main text). $3 \mathrm{~g}$ polyvinyl acetate (PVAC with average molecular weight $170000 \mathrm{~g} / \mathrm{mol}$ ) was dissolved in $20 \mathrm{~mL}$ dimethyl carbonate (DMC) under stirring for $\sim 8 \mathrm{~h}$, which was used as the liquid precursor for the shell. Two kinds of core liquids were used, namely, the deionized water and the aqueous solution with the Au NPs. Note that the glycerol ( $\sim 10 \mathrm{vol} \%)$ was added to the core liquids to reduce the evaporation of water.

For the electrospinning, the temperature and humidity of the chamber enclosed by a metal shell were kept at $\sim 30{ }^{\circ} \mathrm{C}$ and $30 \%$ by the calorifier and dehumidifier, respectively. The liquid core precursor and shell precursor were pumped into two different syringes, which were placed on the syringe pump. The inner and outer diameters of the core nozzle are $0.6 \mathrm{~mm}$ and $0.9 \mathrm{~mm}$, respectively, while the inner diameter of the shell nozzle is $1.3 \mathrm{~mm}$. The shell nozzle is connected to 
a positive electrode of a high-voltage power supply, which generates a dc applied voltage of up to $30 \mathrm{kV}$ that is relative to a grounded collector. The solutions were electrospun at the constant temperature and humidity with an applied voltage of $15 \mathrm{kV}$. The fluid flow rates of the core liquid and shell liquid were $1.12 \mathrm{~mL} / \mathrm{h}$ and $4.38 \mathrm{~mL} / \mathrm{h}$, respectively. The nozzle-to-collector distance was $15 \mathrm{~cm}$ in the experiments. In the electrohydrodynamic process of electrospinning, the liquids are extruded from the spinneret to form the jet. The jet initially extends to a straight line and then undergoes whipping motions due to bending instabilities, which is stretched into fine fibers for the solidification. To fabricate ready-to-use liquid cells for transmission electron microscopy (TEM) observations, the TEM grids (covered by the 20-nm-thick carbon supporting films) was pasted on the roller collector. During electrospinning, the fibers containing a large number of beads (liquid cells) were then directly collected on the TEM grids. The collection duration was fixed at $30 \mathrm{~s}$ after $\sim 2$ min of stable spinning. For investigating the coating effect, the sample surface was deposited with carbon to reduce the charging behavior.

The existence of water in liquid cells. The liquid cell samples were printed on the glass slides with the same parameters shown above. Confocal Raman spectroscopy (Renishaw inVia Qontor) was used to identify the existence of water in the liquid cells. The laser wavelength and power used in the experiments were $532 \mathrm{~nm}$ and $25 \mathrm{~mW}$, respectively. The Raman characteristic line of C-H symmetric stretching of PVAC is $2941 \mathrm{~cm}^{-1}$ and the characteristic peak of $\mathrm{H}_{2} \mathrm{O}$ is $3200-3400 \mathrm{~cm}^{-1} .{ }^{1,2}$

Morphological features of the liquid cells. The liquid cell sample was electrospun on an aluminum foil. A thin gold film was then deposited on the sample surface for $15 \mathrm{~s}$. The surface morphology characterization was carried out by using a scanning electron microscope (NOVA NanoSEM 230).

In-situ visualization of bubbles and their driven NPs motion dynamics. The evolution dynamics 
of the bubbles and Au NPs under the electron beam (e-beam) illumination were in situ visualized by a FEI Talos F200X TEM. The electron dose rate was kept at $10-50 \mathrm{e} \mathrm{nm}^{-2} s^{-1}$. The growth/shrinkage of the bubbles and the motion of the Au NPs in the liquids were simultaneously recorded by a digital camera. The energy dispersive X-ray spectroscopy (EDS) was used to verify the chemicals in the liquid cells.

\section{Supplementary Notes}

Gas pressure of the bubbles. For the bubbles constrained in a columnar liquid cell, the gas pressure $P_{g}$ inside the bubble is,

$$
\begin{aligned}
& P_{g}=P_{l}+P_{\text {surf }} \\
& P_{\text {surf }}=\frac{4 \sigma}{L} \cos \theta
\end{aligned}
$$

where $P_{l}$ is the pressure inside the liquid cell $(\sim 100 \mathrm{kPa}), P_{\text {surf }}$ the Laplace pressure at the gas/liquid interface, $\sigma$ the surface tension of water $\left(7.28 \times 10^{-2} \mathrm{~N} / \mathrm{m}\right),{ }^{3} \mathrm{~L}$ the bubble width and $\theta$ the contact angle (geometric structure shown in Figure S4).

As known from the statistical studies of the main text (Figure 4), the stable widths $L$ of the bubbles were $0.64,0.78$ and $1.10 \mu \mathrm{m}$ for the electron dose rates of 50,20 and $10 \mathrm{e} \mathrm{nm}^{-2} s^{-1}$, respectively. By taking the contact angle (average value of $45^{\circ}$ ) and the above parameters into eqs. (1) and (2), the corresponding gas pressures inside the bubbles were estimated to be 422,362 and $288 \mathrm{kPa}$ at different electron dose rates.

Radiolysis of liquid water. To estimate the $\mathrm{H}_{2}$ concentration in water upon the e-beam excitation, the ESTAR database for electron stopping power is used. ${ }^{4}$ The volumetric production rate is, 


$$
R_{X}=\rho \psi \frac{G(X)}{e N_{A}}
$$

where $X$ means different species of e-beam-induced product, $\rho$ the density of water, $\psi$ the volumetric dose-rate $(\mathrm{Gy} / \mathrm{s}), N_{A}$ the Avogadro number, $e$ (C/electron) the electron charge, and $G(X)$ the empirical parameter regarding the yield of $X$ per $100 \mathrm{eV}$ absorption energy from radiation. The volumetric dose-rate is given by,

$$
\psi=\frac{10^{5} S I}{\pi a^{2}}=10^{5} S R e
$$

where $S=2.78$ ( $\mathrm{MeV} \mathrm{cm}^{2} / \mathrm{g}$ electron) is the density-normalized stopping power obtained from ESTAR database, $I(\mathrm{C} / \mathrm{s})$ the beam current, $a(\mathrm{~m})$ the radius of e-beam, and $R\left[\mathrm{e} /\left(\mathrm{nm}^{2} \cdot \mathrm{s}\right)\right]$ the electron dose rate. ${ }^{5}$

Under the e-beam radiation of water, a certain number of species would be formed including $\mathrm{H}_{2}$ and $\mathrm{O}_{2}$, which could affect the bubbles stability. Normally, the $\mathrm{H}_{2}$ is considered as the main influence factor under typical e-beam irradiation condition. ${ }^{6}$ As the concentration of $\mathrm{H}_{2}$ gradually increases upon irradiation, the volumetric production rate of $\mathrm{H}_{2}$ would decrease, and finally an equilibrium concentration of $\mathrm{H}_{2}$ reaches. The equilibrium concentration is expressed as,

$$
C_{\text {steady-H}}=A_{H_{2}} \psi^{r_{H_{2}}}
$$

where $A_{H_{2}}$ is around $9.3 \times 10^{-7} M(s / G y)^{-r_{H_{2}}}$ and $r_{H_{2}}$ is around 0.44 in water media. ${ }^{7}$ Therefore, the equilibrium concentrations of $\mathrm{H}_{2}$ were calculated to be $0.284,0.386$, and $0.577 \mathrm{mM}$ at 10 , 20 and 50 e $\mathrm{nm}^{-2} s^{-1}$, respectively.

Saturated vapor pressure. According to Henry’s law, the saturated vapor pressure $P_{X}$ is proportional to the concentration $C_{X}$ as 


$$
\mathrm{k}=P_{X} / C_{X}
$$

where $\mathrm{k}$ is the Henry's constant. As known, $C_{H_{2}}$ is $\sim 0.77 \mathrm{mM}$ at $100 \mathrm{kPa}{ }^{5}$ Therefore, by taking the $\mathrm{H}_{2}$ equilibrium concentrations of $0.284,0.386$, and $0.577 \mathrm{mM}$ into eq. (6), the saturated vapor pressures of $\mathrm{H}_{2}$ were estimated to be 37,50 and $75 \mathrm{kPa}$ at the electron dose rates of 10,20 and 50 e $\mathrm{nm}^{-2} s^{-1}$, respectively.

Calculation of Mean Square Displacement (MSD) for nanoparticles. A series of snapshots of nanoparticles motion were extracted from the movie at $t_{0}, t_{1}, t_{2}, \ldots \ldots$, and $t_{N}$ with a time interval of $\Delta \mathrm{t}=0.2 \mathrm{~s}$. The position of nanoparticles at each time point is determined as $r\left(t_{0}\right), r\left(t_{1}\right)$, $r\left(t_{2}\right), \ldots \ldots$, and $r\left(t_{N}\right)$, respectively. The MSD at $t=n \Delta t$ can be obtained by

$$
\operatorname{MSD}(\mathrm{t}=\Delta \mathrm{t})=\frac{\sum_{k=n}^{N}\left[\mathrm{r}\left(t_{k}\right)-\mathrm{r}\left(t_{k-n}\right)\right]^{2}}{N-(n-1)}
$$

where $\mathrm{n}$ is a number from 0 to $\mathrm{N} .{ }^{8}$ 


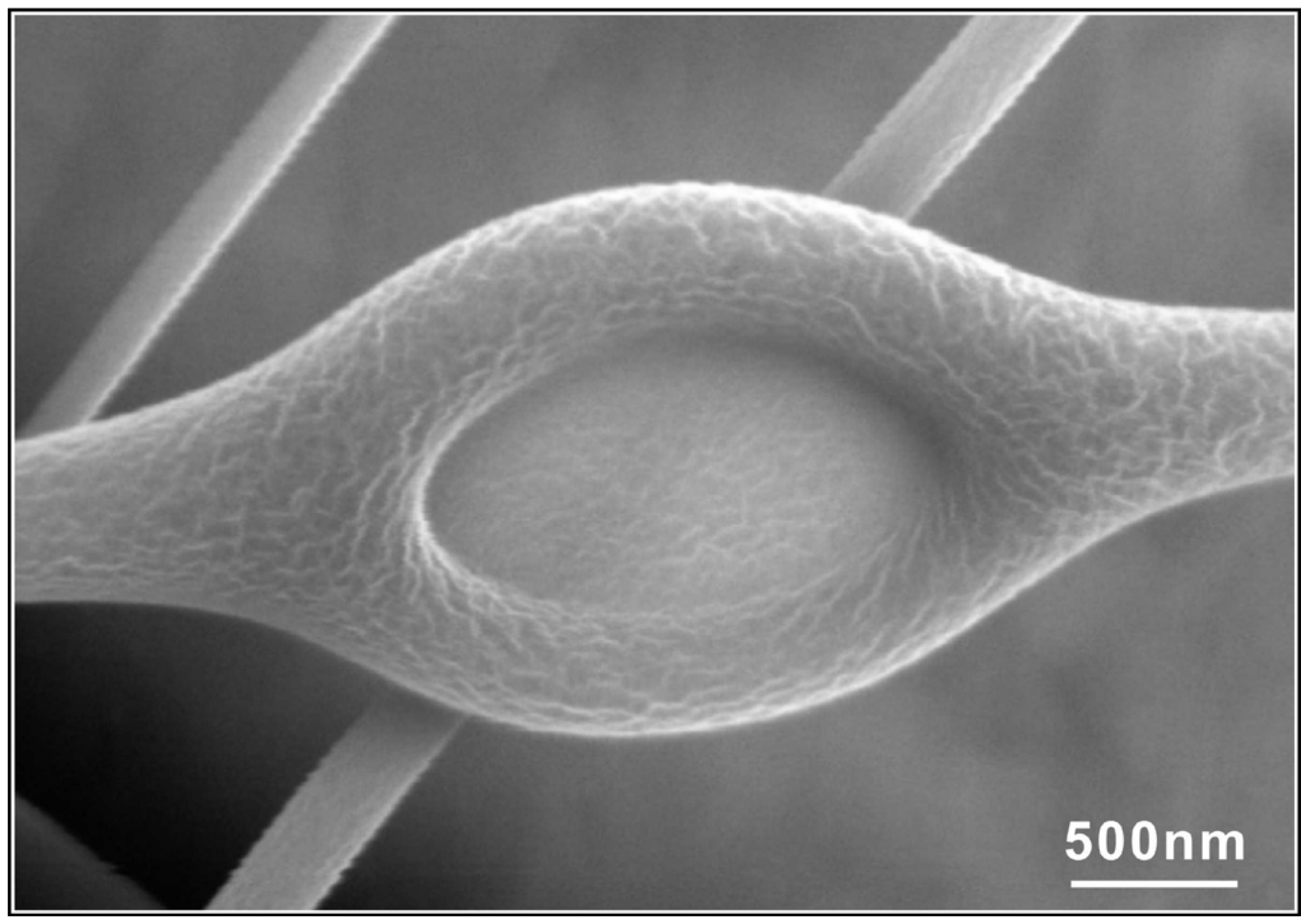

Figure S1. Secondary electron image of a typical liquid cell. 


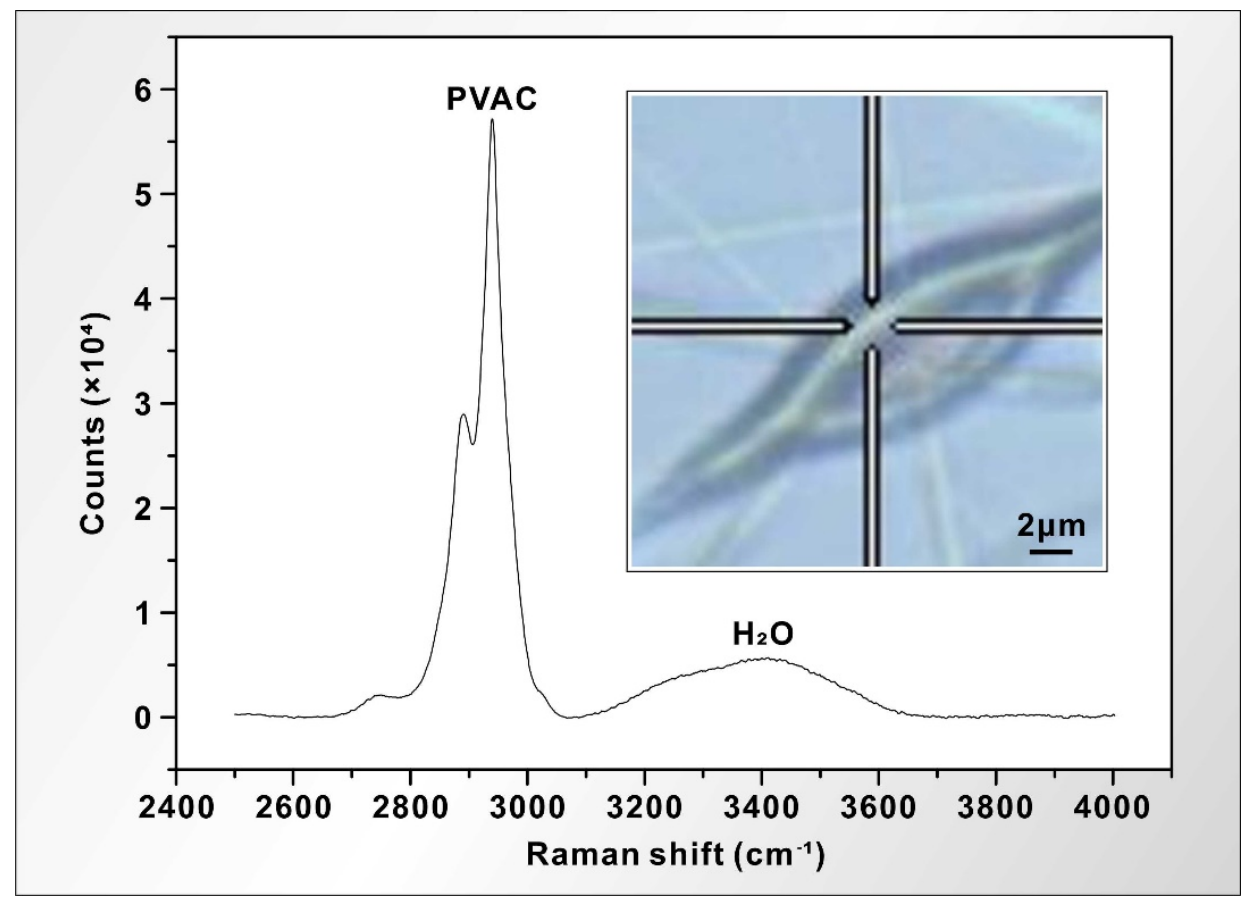

Figure S2. Raman spectrum of the liquid cell. The inset shows the tested position. 


\section{Liquid trace by EDS}

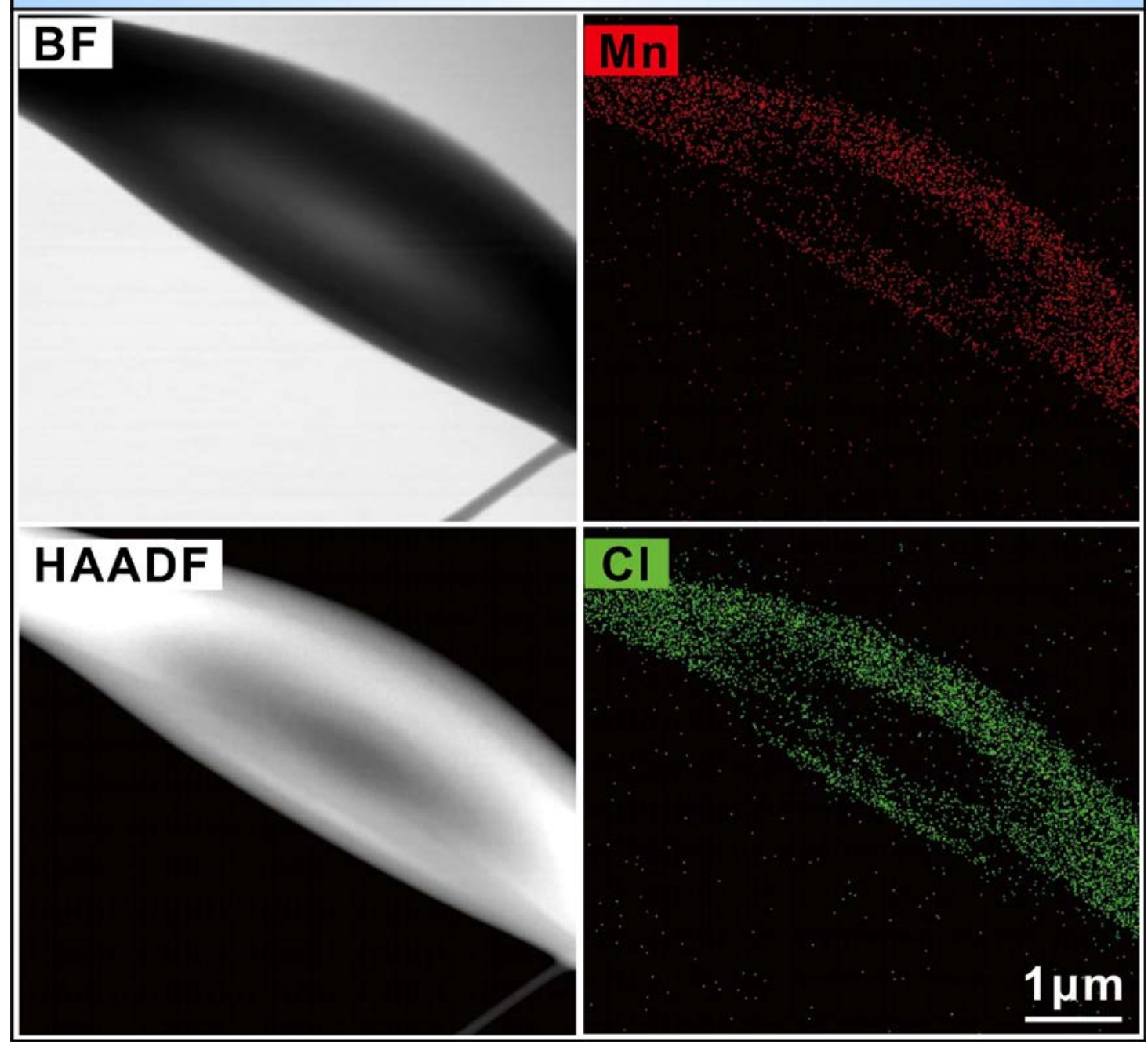

Figure S3. Verification of liquid region inside the liquid cells. (Left column) Bright-field (BF) and high-angle annular dark-field (HAADF) images of a typical liquid cell. (Right column) Energy dispersive $\mathrm{X}$-ray spectroscopy (EDS) analysis of the $\mathrm{MnCl}_{2}$ solution inside the cell. The distribution of $\mathrm{Mn}$ and $\mathrm{Cl}$ elements shows the location of liquid water in the cell. 


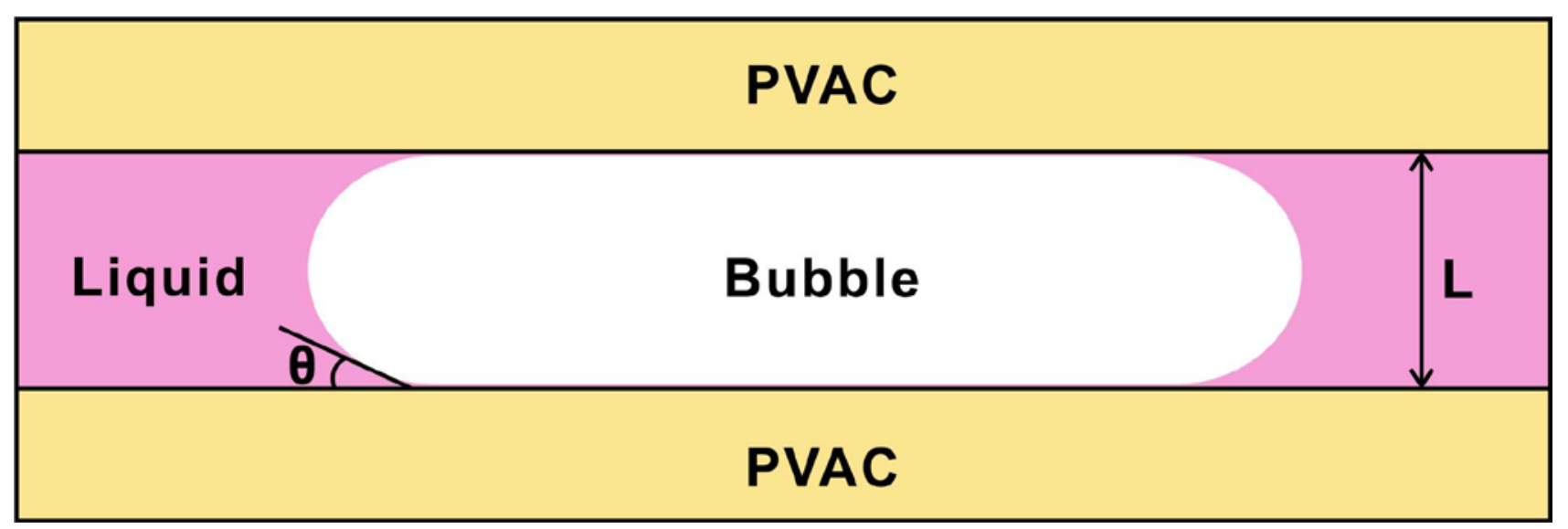

Figure S4. Schematic of a bubble inside the liquid. The liquid is encapsulated by the polyvinyl acetate (PVAC) shell, where a bubble is schematically illustrated inside the liquid cell. The width L of the bubble and the contact angle $\theta$ between the PVAC-gas and the gas-liquid interface are defined. 


\section{Nanoparticles and agglomerate in liquid}

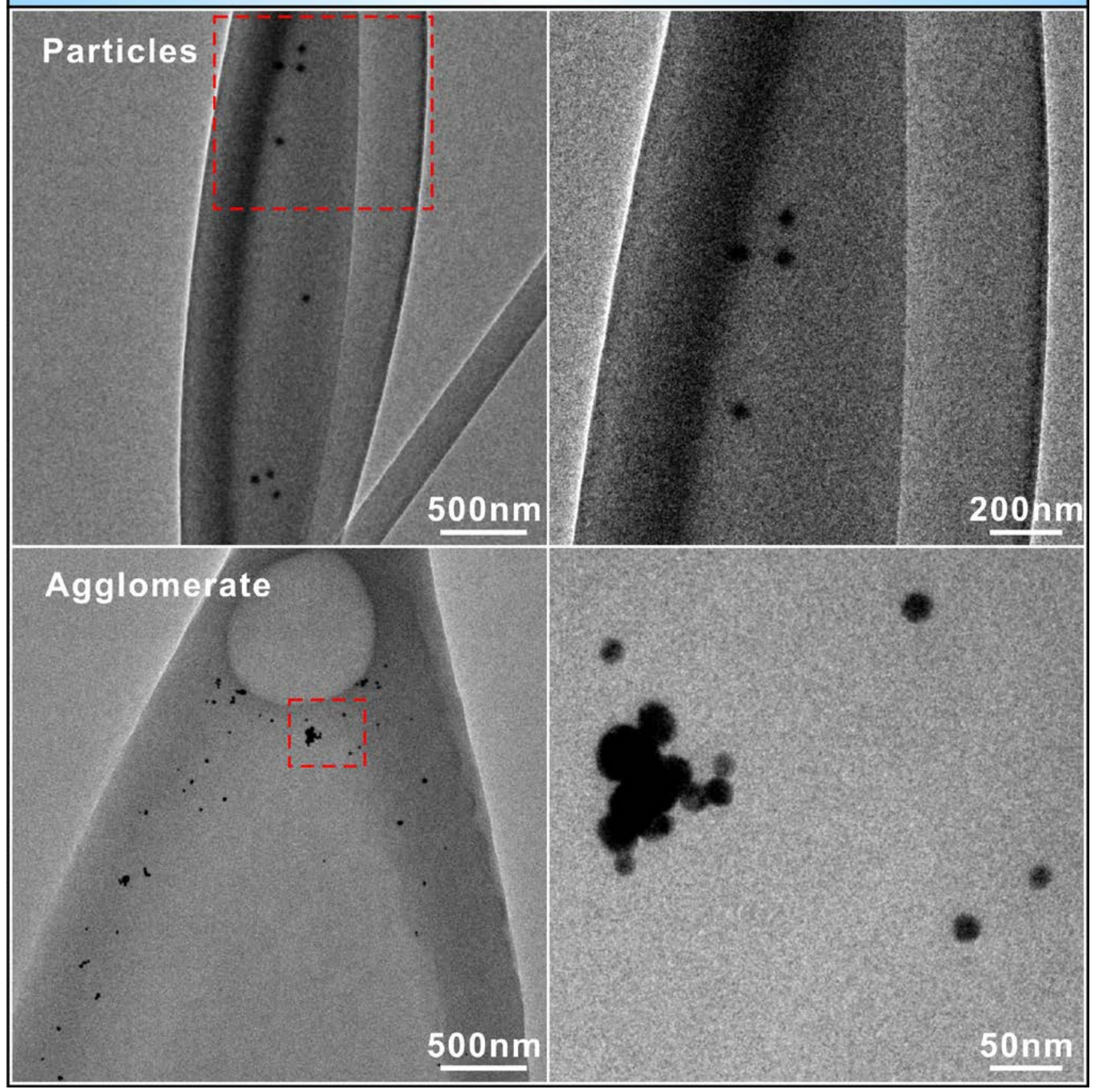

Figure S5. TEM images of singe Au nanoparticles (Top) and the Au agglomerates (Bottom) inside the liquid cells. The right-column images are the enlarged views from the dotted rectangle box shown in the left column. 


\section{Nanoparticles without bubble driving}

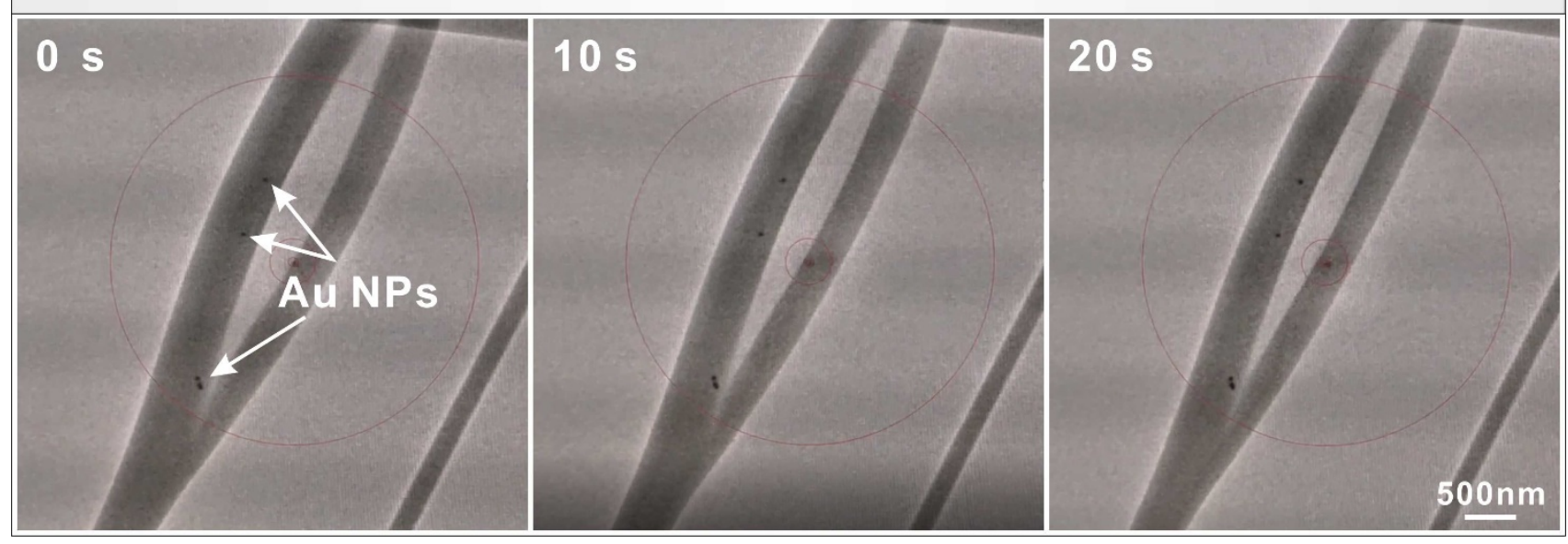

Figure S6. The Au NPs showed no obvious motion when no bubbles existed in the liquid cell. 


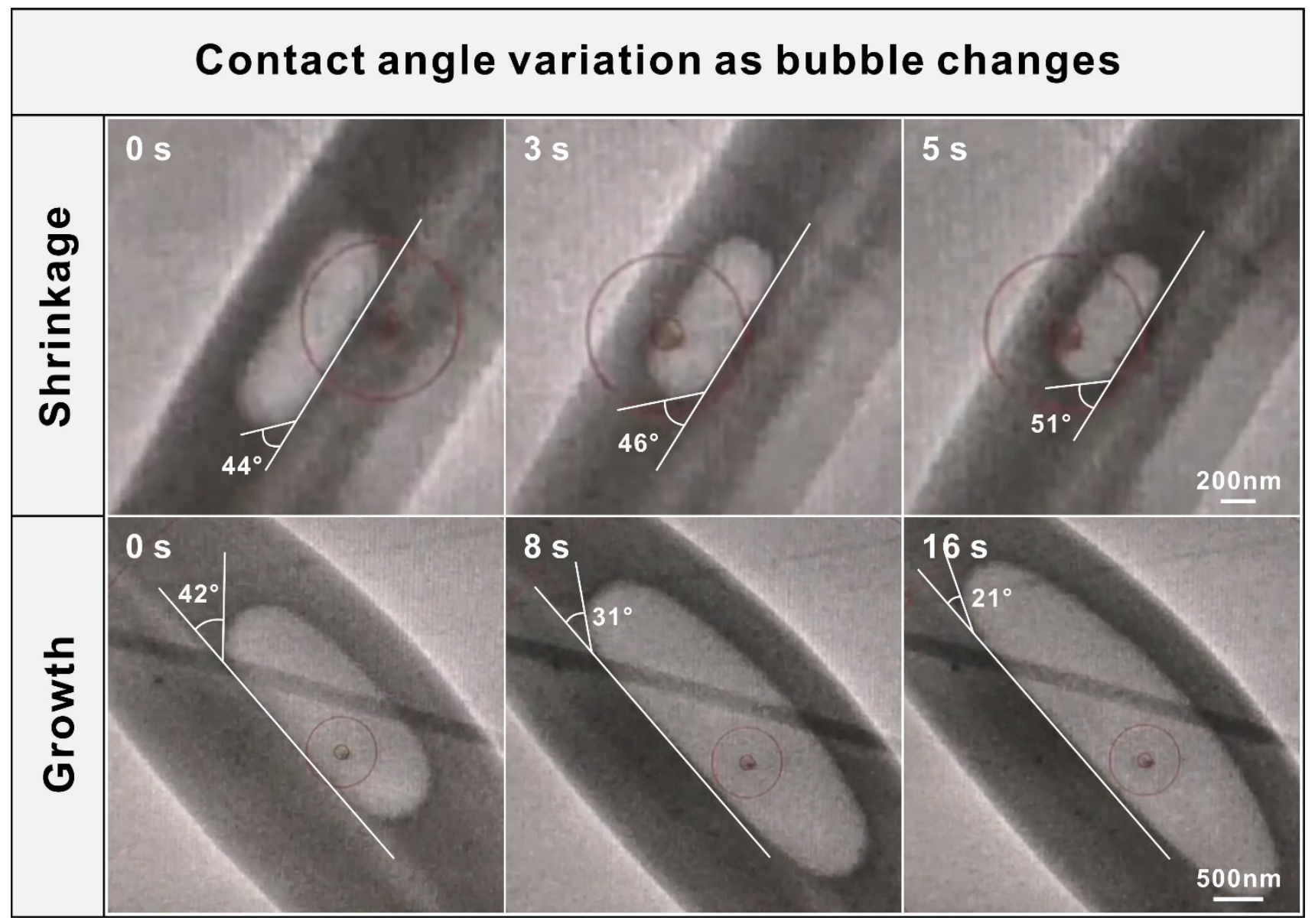

Figure S7. Variation of contact angle during bubble shrinkage and growth. (Top) As the bubble shrinks, the contact angle increases, e.g., from $44^{\circ}$ to $51^{\circ}$ for a bubble shown above. (Bottom) The contact angle decreases when a bubble grows, e.g., from $42^{\circ}$ to $21^{\circ}$ during the growth. 


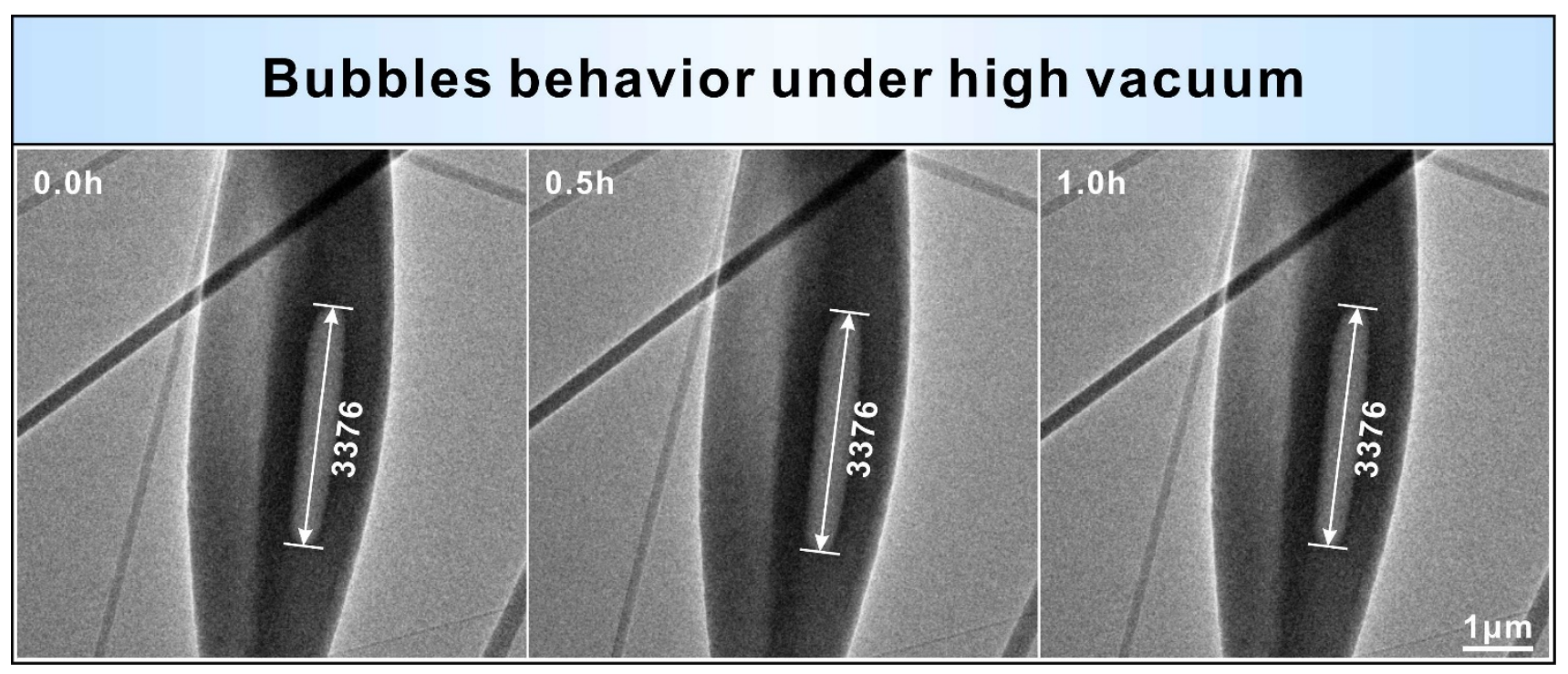

Figure S8. Gas leakage test. Without the e-beam excitation, the bubble can sustain in high vacuum environment for long time. For example, no noticeable change of the bubble size was observed in high vacuum even for $1 \mathrm{~h}$, indicating no gas leakage from the liquid cell. 


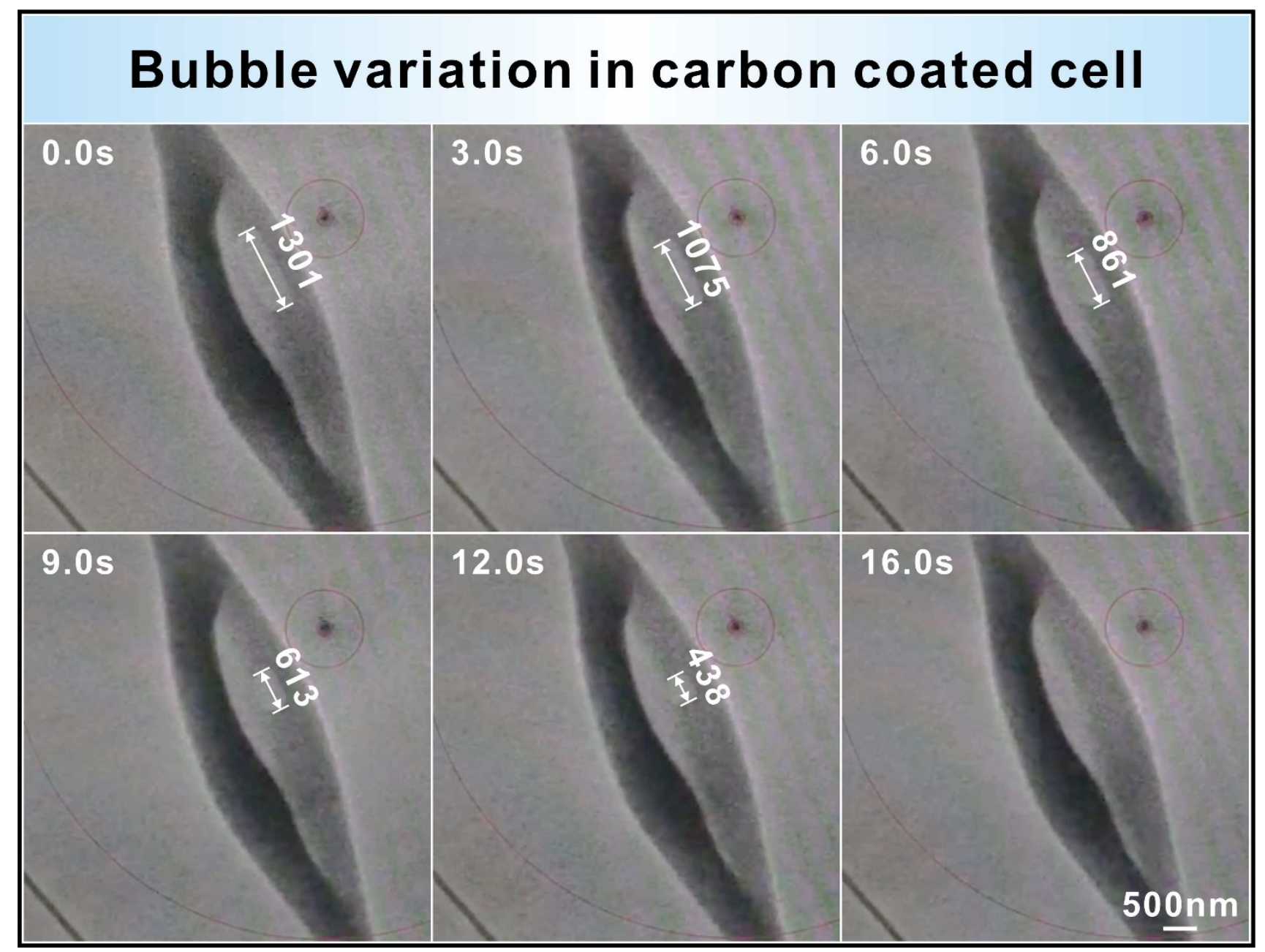

Figure S9. Bubble variation in the liquid cell with carbon coating. A typical example of the coating effect on the bubble variation is presented. A series of snapshots show the bubble shrinkage in the liquid cell with carbon coating upon the e-beam excitation. 
Movie S1. Video of the evolution dynamics of three bubbles upon the e-beam excitation.

Movie S2. Video of the growth behavior of a single bubble.

Movie S3. Video of the shrinking process of a single bubble.

Movie S4. Video of a stable bubble under the electron beam irradiation at double speed.

Movie S5. Video of the coalescence behavior of three bubbles inside the same liquid cell.

Movie S6. Video of the bubble-driven motion of single Au particles.

Movie S7. Video of the bubble-driven motion of Au agglomerates.

\section{References:}

1. Schabel W.; Ludwig I.; Kind M. Measurements of concentration profiles in polymeric solvent coatings by means of an inverse confocal micro Raman spectrometer-initial results. Dry. Technol. 2004, 22(1-2), 285-294.

2. Starzak M.; Mathlouthi M. Cluster composition of liquid water derived from laser-Raman spectra and molecular simulation data. Food Chem. 2003, 82(1), 3-22.

3. Takamura, K.; Fischer, H.; Morrow, N. R. Physical properties of aqueous glycerol solutions. J. Petrol. Sci. Eng. 2012, 98-99, 50-60.

4. Suplee, C. Stopping-power \& range tables for electrons, protons, and helium ions. Nistir 2009, http://www.nist.gov/pml/data/star/index.cfm/.

5. Grogan, J. M.; Schneider, N. M.; Ross, F. M.; Bau, H. H. Bubble and pattern formation in liquid induced by an electron beam. Nano Lett. 2014, 14 (1), 359-364. 
6. Pastina, B.; LaVerne, J. A. Effect of molecular hydrogen on hydrogen peroxide in water radiolysis. J. Phys. Chem. A 2001, 105 (40), 9316-9322.

7. LaVerne, J. A.; Pimblott, S. M. Scavenger and time dependences of radicals and molecular products in the electron radiolysis of water: Examination of experiments and models. J. Phys. Chem. 1991, 95 (8), 3196-3206.

8. Kheifets, S.; Simha, A.; Melin, K.; Li, T. C.; Raizen, M. G. Observation of brownian motion in liquids at short times: Instantaneous velocity and memory loss. Science 2014, 343 (6178), 14931496. 